\title{
El cáncer, un desafío común. De la percepción pública a la responsabilidad social
}

\author{
Sonia Ponce de León Villafuerte \\ Centro de Inmunología Molecular (Cuba) \\ Yamilé Ferrán Fernández \\ Rayza Portal Moreno \\ Facultad de Comunicación, Universidad de La Habana
}

\section{Palabras clave \\ Comunicación en salud, percepción pública, responsabilidad social, cáncer, \\ Centro de Inmunología Molecular.}

\begin{abstract}
Resumen
El presente artículo se objetiva a partir de una investigación de carácter empírico exploratoria interesada en un diagnóstico primario sobre el estado de configuración de la percepción pública en el sector salud en torno al fenómeno del cáncer en Cuba; procurando otorgar voz reflexiva a investigadores del nivel terciario, a pacientes y familiares, y tomando al Centro de Inmunología Molecular (CIM) como institución medular para este abordaje, desde un enfoque de responsabilidad social.

A través de la pesquisa se explicitan los vacíos y fracturas en torno a relaciones y responsabilidades compartidas tanto hacia los sectores involucrados del ámbito científico y de la salud, las instituciones como los sujetos/pacientes, así como el carácter estratégico de la comunicación en salud como expresión de una comunicación de las ciencias de servicio público. De igual modo, desde la visión crítica de los actores pulsados, preponderó la legitimidad de fomentar una percepción pública coherente a partir de la promoción, educación y prevención de salud, con la requerida sistematicidad y horizontalidad social que permita que ella trascienda, más allá de los saberes e informaciones más mediáticas que acompañan al reconocimiento público sobre la enfermedad, al cambio actitudinal de los sujetos involucrados y sus familias, en un contexto país donde el cáncer constituye la primera causa de mortalidad en adultos de ambos sexos.
\end{abstract}




\section{Cancer, a common challenge. Public perception of social responsibility}

\author{
Key words \\ health communication \\ public perception; \\ scicial responsibility; cancer.
}

Abstract

This work is based on a study of public perceptions of the phenomenon of cancer in Cuba, who was interested in uncovering the reflective level on this subject from the perspective of stakeholders, taking the Center of Molecular Immunology (CIM) as a core institution this approach.

In the investigation are exposed relationships and responsibilities relevant sectors, institutions and individuals, the scientific, health and communication. Similarly, in the voice of the actors interviewed it prevailed promote the legitimacy of public perception from a promotion, education and prevention in health coherent and systematic that transcends beyond the knowledge that accompany the recognition of the disease, attitudinal change the subject involved and their families.

\section{Cómo citar el artículo}

Ponce de Léon, S.; Ferrán Fernández, Y.; Portal Moreno, R. (2016). El cáncer, un desafío común. De la percepción pública a la responsabilidad social. Revista de Comunicación y Salud, Vol. 6, pp. 43-54.

DOI: http://doi.org/10.35669/revistadecomunicacionysalud.2016.6.43-54 


\section{Introducción}

La población cubana estructura desde hace más de dos décadas su comportamiento desde una tendencia ascendente al envejecimiento, trayendo consigo, entre otras implicaciones, la confirmación de enfermedades crónicas como la hipertensión y la diabetes, si bien con mayor énfasis en el último lustro la tendencia se ha intencionado hacia una enfermedad como el cáncer, considerado por muchos un flagelo global; manifestándose de manera general, en adultos mayores de 50 años. De acuerdo a datos preliminares de cierre del 2014, de la Oficina Nacional de Estadística e Información (ONEI), las cifras de mortalidad en la población rebasan los más de 22.000 casos, lo cual sitúa la enfermedad en la primera causa de muerte en Cuba. Por esta razón médicos y científicos cubanos han centrado su atención en este fenómeno generando nuevos productos y tratamientos en busca de mejorar y extender la calidad, y esperanza de vida de este tipo de pacientes. Actualmente el estimado de prevalencia del cáncer es de 135.000 enfermos, expresándose en cada uno de ellos de diversas formas, en dependencia del tipo de cáncer y de la predisposición de cada organismo.

El cáncer es una enfermedad que tiende a la cronicidad, es decir, que dura en el tiempo y debe ser controlada por años. Ello induce a plantearse desafíos para el Estado y sus entidades de servicio público y presupuestario; para el campo de la ciencia y sus instituciones productoras, y para la sociedad civil y sus estructuras básicas con encargos educativos y de reproducción. Con puntualidad el fenómeno se hace más vulnerable en el eslabón paciente/ en cuanto a temas como alimentación, medicamentos, condiciones de vida e higiene, efectos adversos de las terapias existentes, su control y a qué nivel de salud (primario o secundario) debe asistir para el tratamiento general.

Tomando en cuenta que se trata de una enfermedad compleja, con resultados estadísticos anuales que muestran una incidencia de 31.757 casos nuevos; considerando que la población cubana tiene un limitado acceso a Internet, y una limitada promoción de salud para el cáncer en los medios de comunicación dirigidos al gran público, se impone la necesidad de repensar alternativas verdaderamente accesibles que estén en función de suscitar una información pública coherente, actualizada y sistemática, como plataforma de efectiva promoción, prevención y educación en salud.

Es pues desde la responsabilidad institucional y empresarial con alcance social que le atañe al Centro de Inmunología Molecular (CIM, entidad de ciclo cerrado: investigación-desarrollo, producción, comercialización, verticalizada a la investigación del cáncer y otras enfermedades del sistema inmune), que se verifica un interés por actualizar, desde un diagnóstico previo, la información y los saberes que a nivel público se manejan en torno al cáncer, sus tipologías, efectos, terapéuticas vigentes, entre otras disquisiciones, así como a suscitar una reflexión crítica sobre la temática y sus vacíos según la percepción de la comunidad científica de investigadores.

Como enfoque novedoso al interior de las prácticas investigativas que distinguen esta producción científica, se buscó poner en diálogo los procesos de percepción pública con los de responsabilidad institucional, otorgándole, a partir de los resultados del estudio y la triangulación propuesta, una visión crítico reflexiva a la actuación del Centro de Inmunología Molecular que le permita dimensionar, robustecer y comprometer con mayor profundidad su misión de proveedor de conocimientos, de saberes y de opinión pública hacia la sociedad, la comunidad y la ciudadanía, desde un enfoque holístico del tema cáncer y en compromiso deliberado con una efectiva educación, promoción y prevención de salud. Asimismo, la exploración se interesó por identificar las vías y canales que hoy actúan y las que han de constituirse en deber ser de esta tipología infocomunicativa, al tiempo de prescribir las articulaciones estratégicas desde la responsabilidad social y científica para una puesta en 
escena pública por parte del CIM en el abordaje socia, sectorial y comunitario del tratamiento cáncer y su gestión como productor de vacunas en Cuba.

Como premisas de la investigación, se partió de los siguientes supuestos:

- Acciones con alto compromiso, responsabilidad y contribución social como lo es el tratamiento a las enfermedades de incidencia en la morbilidad como el cáncer, reclaman el concurso multidimensional, transdisciplinar e interinstitucional de todos los organismos y agentes que a nivel de sociedad cubana actual concurren con encargos explícitos o implícitos vinculados con esta enfermedad. Solo desde una gestión articulada y articuladora de todos estos niveles, planos de acción y organizaciones, podrá rebasarse la mirada sectorial y emprenderse realmente una eficiente y sostenida labor de comunicación científica pública, promoción y educación para la salud.

- La percepción pública en torno a esta enfermedad, su impacto social, sus terapéuticas, pasan necesariamente por el tamiz de los medios masivos de comunicación, y de aquella representación noticiosa, opinática, reflexiva y de saberes en general que ellos sean capaces de poner en la agenda pública; asimismo les corresponde, esté o no explícito y sea o no coordinado con las entidades prescriptoras, poner en circulación los contenidos relativos a la educación, promoción y prevención de salud, proceso para el cual son decisivas las instancias de salud (en cualquiera de sus tres niveles), en un esfuerzo mancomunado con los mass media; si bien intervienen otros factores y mediaciones a nivel estratégico como las relativas a la sensibilidad que a nivel institucional y privado sean capaces de fomentar en la ciudadanía dos figuras clave: la escuela y la familia, respectivamente.

- El Centro de Inmunología Molecular (adscrito al Grupo BioCubaFarma) se inserta en este ámbito decisional con condiciones inmejorables para promover, desde la autoridad cognitiva y científica que le otorgan su prestigio institucional, la calidad de sus procesos de innovación y producción, así como las competencias de su capital humano, un debate público y una convocatoria social en aras de aunar voluntades institucionales en torno al tratamiento que el tema reclama. Es desde un ejercicio consecuente de Responsabilidad Social, que entidades como estas deben trascender sus encargos más inmediatos para asumir roles más proactivos.

Desde el asiento teórico, el estudio sistematizó los enfoques conceptuales más actualizados, a partir de autores latinoamericanos y europeos en torno a la percepción pública de las ciencias, de la salud, la responsabilidad social, en deliberado desplazamiento desde las visiones corporativas hacia lo público e institucional, al tiempo de ponderar las nociones de prevención, educación y promoción de salud que han de actuar como transversalidades y mediaciones.

Es entonces este análisis, el que permite comprender cómo el proceso de percepción pública está dado por las ideas que poseen las personas acerca del mundo que los rodea, las creencias y actitudes que se van construyendo poco a poco a partir de lo que se experimenta cotidianamente, de la información disponible, de los conocimientos, valores y modelos de pensamiento que son transmitidos a través de los medios de comunicación, la educación, la historia y la tradición: el conocimiento se construye de manera individual y social (Jara \& Torres, 2011).

Si bien en el mapa de instituciones que inciden y tributan a la percepción pública conviven instancias cuyo espacio de actuación es más privado (la familia, los grupos de pertenencia...), son básicamente los desempeños públicos los que interesan a los decisores políticos a la hora de estructurar y gestionar con alcance social programas relativos a la salud de grandes conglomerados. En esta construcción de las opiniones acerca del mundo, como se ha sugerido, los medios de comunicación tienen una influencia tal que bajo determinadas coyunturas pueden subvertir y desplazar del sentido deseado la labor que realizan determinadas instituciones. Históricamente han tenido una ascendencia muy fuerte 
en las audiencias, y si bien, el sujeto social del siglo XXI como agente activo de una sociedad global interactiva ya dejó atrás con mucho la visión apocalíptica e hipodérmica de creer en todo cuanto le dicen los medios, allí donde se produzcan vacíos y áreas de desatención en las redes cuyo encargo ha de ser informarlo desde la prevención, fructificará inexorablemente una visión interpretativa parcializada, fragmentaria o tergiversada de la problemática de salud.

En este caleidoscopio adquiere pues relieve, de una parte el incentivo a las competencias cognitivas de los sujetos, y su subjetividad, a través de una sistemática labor de educación y promoción de salud, de otra la fiscalización desde las políticas públicas (de salud, científicas, educativas y mediáticas, fundamentalmente) de los comportamientos institucionales en torno a una responsabilidad social común coherente y proactiva, en cuyo frente han de ubicarse los medios masivos de comunicación.

Desde luego que en tal complejo entramado no pueden desconocerse aspectos como el habitus $^{1}$ que ha venido cultivando determinada sociedad sobre los sujetos; las competencias que han de asignársele coherentemente al aparato escolar en el cometido de generar desde edades tempranas la sensibilidad por una salud plena, al tiempo de incluir a la familia como repositorio de saberes y de valores a la cual el individuo regresa una y otra vez para confirmar, fijar y retribuir lo aprehendido en torno a patrones y apropiaciones externas.

De tal suerte, dígase que toda percepción pública es por esencia una sumatoria no mecánica, y sí articulada de informaciones, saberes, conocimientos, cuyas fuentes de emisión son múltiples y diversas, tanto por su jerarquía y encargo social, como por la proximidad y/o subordinación respecto a las políticas públicas.

Como se acotaba con anterioridad, aunque el sujeto contemporáneo dispone en su condición de receptor activo (alfabetizado, informado, instruido mediáticamente hablando) de mayor poder discriminatorio para seleccionar de cuánto dicen los medios lo más compacto y veraz, en materia de salud, siempre serán las entidades de servicios directos (centros de salud y su personal asociado) y las instituciones de producción científica las que gocen de mayor autoridad y notoriedad como fuentes, de ahí que sea siempre necesario capacitar para la comunicación de salud a los profesionales de estos sectores públicos, en aras de hacerlos partícipes de herramientas efectivas que permitan el diálogo y la comunicación hacia el sujeto final de esta larga cadena, sin desestimar los aspectos científicos y la veracidad, como tampoco hiperbolizando el discurso especializado.

En la literatura consultada acerca de la percepción pública y sus complejidades, es recurrente observar una reflexión en torno al divorcio entre los intereses de los sujetos sociales y las agendas que construyen y oficializan los medios. Refiere el académico mexicano de la UNAM, Dr. José Antonio de la Peña, en una suerte de paneo comentado sobre la actualidad de la percepción de la ciencia en ese país, cómo a lo largo de la historia el poder de los medios ha crecido de manera impresionante, sin embargo el público en general aún tiene ideas vagas en lo que respecta a la ciencia y esto ha dependido en gran medida en la poca profundidad de los estudios que se realizan sobre intereses, actitudes y niveles de comprensión del público ante los diferentes fenómenos a los que se enfrenta cada día, por lo que en este contexto se van dejando en la información que se quiere trasmitir, evidentes brechas o como en muchos casos, falsas informaciones (de la Peña, 2005).

\footnotetext{
${ }^{1}$ Concepto planteado por el destacado sociólogo francés Pierre Bourdieu que refiere mecanismos de orientación que hacen que los sujetos inconscientemente se orienten a aquellas prácticas para las cuales se sienten que fueron hechos de acuerdo a sus clases sociales, género, raza, estatus, etc.
} 


\section{Objetivo}

Desde tales lógicas teóricas y conceptuales, la investigación entonces se objetivó a partir de un propósito científico clave: analizar el estatuto teórico y funcional que diversos actores involucrados en el impacto social del cáncer en Cuba le otorgan a la percepción pública de esta enfermedad.

\section{Metodología}

La pesquisa se concibió con un hálito exploratorio, como un estudio de caso único global, otorgando valía en el orden empírico a técnicas como la encuesta (a pacientes y familiares); el análisis documental cualitativo; análisis mediático (contenidos de programas televisivos y radiales sobre cáncer); la observación de las dinámicas de diagnósticos y terapias postoperatorias en centros asistenciales especializados y el "focal group". En el caso de la entrevista en profundidad, asumida intencionalmente como la técnica central de recogida de información, que permitiría pulsar la interpretación de los sujetos, se abrió hacia distintos grupos de actores, todos con alta incidencia en la configuración de la opinión pública más sectorial (especialistas de primer y segundo grado de los centros al tiempo que profesores e investigadores): Hospital Docente Clínico Quirúrgico Hermanos Ameijeiras, el Centro de Investigaciones Médico-Quirúrgicas (CIMEQ), así como el Instituto Nacional de Oncología y Radiobiología (INOR, que funge como centro rector o de referencia del Programa Nacional de Oncología del Sistema Nacional de Salud Pública en Cuba), son además entidades cuyos staff especializados acumulan años de experiencia y trabajo conjunto con el CIM, tanto en las fases de ensayos clínicos como en los tratamientos que incluyen la aplicación de anticuerpos monoclonales y vacunas producidas por la institución (CIMAher y CIMAvax) así como a directivos institucionales del CIM (5 entrevistados).

En cuanto a la encuesta y conversatorio con los pacientes y familiares, se le aplicó a 50 sujetos, durante los procesos de atención médica.

\section{Resultados y discusión}

Del $100 \%$ de los pacientes de cáncer encuestados, el $25 \%$ refirieron poseer un conocimiento amplio y profundo de la enfermedad antes de serle detectada, mientras que el $40 \%$ se describió más o menos actualizado, en tanto un $35 \%$ adujo que no conocía absolutamente nada del tema. Sin embargo es apreciable y cabe destacar que se identificaron en estos sujetos como un elemento común y mayoritario, el hecho de que las formas por las cuales obtuvieron conocimiento sobre la enfermedad, estuvieron dadas por experiencias de familiares cercanos que padecieron de cáncer y solo apenas unos pocos por sus profesiones; llama poderosamente la atención que de la totalidad de los pacientes encuestados, ninguno identificó a los medios masivos de comunicación, ni ninguna otra vía o canal público como fuente de información primaria.

\subsection{La atención médica (primaria, secundaria y terciaria) como centro del análisis}

El instrumento aplicado se interesaba por identificar en el Sistema Nacional de Salud, cuál era la instancia que estos pacientes asumían como más cercana, efectiva y cuál no, partiendo de la racionalidad de que es el escalón de atención primaria el encargado de promover el diagnóstico y redirigir al paciente a partir de una consulta general. En tal sentido de un $100 \%$ de pacientes solamente el $10 \%$ tuvo su primer vínculo con la enfermedad con la atención médica primaria (Médico de familia y Policlínico), un $40 \%$ con la atención secundaria (Hospitales Generales) y un 50 \% con la atención terciaria (Instituciones especializadas). Estos datos evidencian como la población se dirige directamente a hospitales generales o instituciones especializadas, en busca de un diagnóstico más rápido y efectivo, lo que demuestra como la labor de la atención primaria, aun cuando es extensiva en todo el país y se ramifica hacia la totalidad de las localidades por más apartadas que 
estén de los centros urbanos, no funge con la debida efectividad porque no siempre existen las condiciones materiales para ello, y median otros factores como la estabilidad del recurso humano, la superación necesaria del personal para que ese proceso tan decisivo del primer vínculo se produzca desde esta instancia.

A juicio de los profesionales expertos adquiere especial valía la preparación de los profesionales de la salud, su actualización, así como la sistematicidad y profundidad tanto de la promoción de salud como de la fase de prevención de una enfermedad. En el caso de la lucha contra la neoplasia se trataría de una detección temprana o en el mejor de los casos, de estudios y autochequeos frecuentes por parte del propio paciente (prueba citológica, autoexamen de mama, como los más recurrentes).

Una voz autorizada, como la Dra Martha Osorio, del INOR, pone el acento en la urgencia de una mayor vinculación entre centros de atención terciaria y los niveles primarios y secundarios, al tiempo de exigir la actualización permanente de aquellos profesionales que colocados en los primeros eslabones de la detección temprana de la enfermedad, redirigen en forma acertada y sin dilación al paciente hacia instituciones como el Instituto Nacional de Oncología y Radiobiología, con todos los recursos y disponibilidades para cubrir eficientemente cualquier tipo de exigencia.

\subsection{De la atención a la promoción de salud, un camino de ida y vuelta}

Sobre las competencias atribuidas a los diferentes niveles de atención (primaria, secundaria y terciaria) en la Promoción de Salud, saltan a la vista insuficiencias que conviven con otros muchos elementos de carácter positivo, pero que al cabo no allanan de forma lineal, efectiva y expedita el camino hacia prestaciones de servicios asistenciales verdaderamente de calidad y oportunidad en el tiempo. Según la Dra. Tania Crombet inciden: poco dominio del tema de los profesionales de la salud; pobre conocimiento en la población de los hábitos de vida con impacto en la reducción de mortalidad; poco conocimiento en programas de pesquisa activa de PSA tales como mamografía, serología, sangre oculta en heces fecales; insuficiente control de la implementación de los programas de pesquisa, así como la prevalencia de un sistema de salud no preparado para garantizar las pruebas confirmatorias de cáncer, dada la positividad de la pesquisa activa.

Asimismo la experta apunta fortalezas que a modo de generalidades respaldan ciertos atributos intrínsecos del Sistema de Salud cubano: gran cantidad de profesionales trabajando en la atención primaria, secundaria y terciaria del cáncer, que como norma tributa a una relación en extremo favorable de personal de salud por habitante; de igual forma la disponibilidad de pruebas para el diagnóstico temprano de la enfermedad de algunas neoplasias, así como una población globalmente instruida, capaz de comprender y sensibilizarse con las campañas de promoción de salud.

Según refiere el investigador y Director de Calidad del CIM, Antonio Vallín García, "las mayores fortalezas (mejor expresadas en oportunidades) están en el sistema primario, aunque se usa de manera insuficiente, en tanto las mayores debilidades están en la combinación multifactorial dada por la poca accesibilidad a la información por parte de la población, una infraestructura asistencial depauperada y fraccionada, unido a la poca preparación y diseminación de la información entre el sector profesional a nivel nacional, en coincidencia con afectaciones en términos presupuestarios del sistema que permitan revertir esta situación".

De tal suerte, coinciden expertos médicos y científicos, en que la información es la punta del iceberg, en la medida en que no fluya como corresponde la información científica, médica y especializada entre el sector profesional se afectará inexorablemente y en efecto cadena, la información pública.

\subsection{Expectativas y valoraciones sobre la promoción de salud}

Se constató una sensibilidad común en torno a vacíos, discontinuidades, acciones de bajo impacto y débiles articulaciones; todo lo cual favorece la reproducción de posturas de 
fragmentación, que tienden a alimentar presunciones limitadas y no siempre certeras sobre la enfermedad en la población, a falta de una estructuración sólida, sistémica, sostenida en el tiempo tanto de información massmediática como de promoción enfocada a la prevención y a más largo plazo a la educación para una salud más plena, basada en un cambio actitudinal y una visión compartida entre todos los agentes concurrentes.

En el criterio generalizado de cientistas y médicos, los programas nacionales (como lo es el Programa Integral para el Control del Cáncer en Cuba) que son definitivamente una apuesta coherente por la eficiencia médica, la detección temprana de determinadas enfermedades y en sí mismos presuponen la interrelación de agentes decisionales, políticas y pacientes, desde su propia concepción están sesgados por un enfoque demasiado sectorial y curativo, en esta medida necesitados de ser repensados bajo una filosofía más participativa, educativa y de promoción de salud, o de lo contrario, habilitar otros programas que en cascada permitan cubrir tales vacíos.

Desde el orden conceptual, es impensable una coherente promoción de salud, al margen de una educación por y para la salud, que no descanse en roles sectoriales e institucionales acotados sino que sea pensada e implementada desde las propias racionalidades y encargos de la familia y de la escuela.

Bajo estos derroteros, la prevención de salud no sería entendida como el "arte de corregir" en tanto medio de "buen encauzamiento", y que mal aplicado pudiera tomarse por peligroso, suscribiría sospechosamente un Foucault. Amén de lo fecundo de una discusión en torno a los límites y raseros que ha impuesto la realidad clasista eurocéntrica y occidental al término, el buen encauzamiento de una prevención efectiva pasaría indefectiblemente por sustentar políticas de salud basadas en buenas prácticas de prevención desde la participación real de todos los sujetos sociales, con alcance a estilos de vida saludables y equitativos socialmente, a generar comunidades y prácticas locales de abajo hacia arriba que permitan descolocar el esquema "bancario" y paternalista en torno a un Estado benefactor y omnisciente, y propiciar esfuerzos de sostenibilidad/sustentabilidad endógena.

\subsection{Tratamiento mediático/organizacional}

En cuanto al tratamiento mediático en los medios de masas se soslaya el valor del abordaje testimonial de la información, lo que en materia de promoción de salud históricamente ha demostrado ser en extremo viable para persuadir y sensibilizar a sectores vulnerables y potenciales ante una determinada enfermedad, así como espacios públicos de debate.

\subsection{Articulaciones estratégicas}

El CIM en su interior cuenta con un alto nivel de actividad científica y diseminación de la información, de igual forma se proyecta hacia afuera para la comunidad médica y científica, no siendo así para la comunidad o la sociedad en general. Aun cuando es una organización suficientemente sensibilizada por sus trabajadores en cuanto a misión y visión, en ellos no queda del todo explicitado su responsabilidad para con la sociedad, desde el concepto de proveedores de conocimiento.

El estudio permitió constatar asimismo, que los directivos y especialistas de CIM, sustentan una visión orgánica, sistémica, veraz y crítica que los convierte en agentes con formidables potencialidades para hacer transcender esa Responsabilidad Social Institucional con mucho mayor fuerza sobre la ciudadanía en su conjunto, sobre la comunidad más inmediata al centro y sobre el propio campo científico y asistencial al cual se vincula por su objeto social, su misión y su visión. 


\section{Conclusiones}

El cáncer adquiere por su impacto en la población mundial niveles inusitados, más allá de demarcaciones etarias, raciales, culturales o de cualquier otro tipo. En Cuba, se expresa estadísticamente, desde hace dos años, como la primera causa de muerte, desplazando a las enfermedades tradicionales.

La investigación permitió constatar una sensibilidad común en torno a vacíos, discontinuidades, acciones de bajo impacto y débiles articulaciones; todo lo cual favorece la reproducción de posturas de fragmentación, que tienden a alimentar presunciones limitadas y no siempre certeras sobre la enfermedad en la población, a falta de una estructuración sólida, sistémica, sostenida en el tiempo tanto de información mass mediática como de promoción enfocada a la prevención y a más largo plazo a la educación para una salud más plena, basada en un cambio actitudinal y una visión compartida entre todos los agentes concurrentes.

Asimismo, compromete entre los actores pulsados el consenso en torno a una promoción de salud deficitaria, la cual a pesar de estar incluida y establecida en el Programa Integral para el Control del Cáncer, a nivel de país, adolece de una estrategia integral de tratamiento, de una infraestructura que la soporte coherentemente, así como de una divulgación constante y de gran alcance; desde canales y medios que se activen en aras de lograr una mayor participación de la sociedad en su conjunto, que conllevaría a incidir no solo sobre el paciente real sino sobre el potencial.

En el plano de la divulgación mediática y pública de factores de riesgos más frecuentes en la sociedad cubana, puede con certeza afirmarse, que ha sido fundamentalmente enfocada a sensibilizar a la población respecto a enfermedades de trasmisión sexual; otras patologías como el cáncer han sido objeto menos abordados por estas políticas y prácticas. En consonancia con esta situación, es usual que los ciudadanos no dominen los llamados "síntomas de alerta de cáncer", lo que ciertamente ha estado mediado también por la limitada accesibilidad a la información y a su vez condicionado no solo en el conocimiento que puedan adquirir en el tiempo, sino, en el cambio de actitud que esto pueda conllevar en tanto alimentación e higiene saludables, disposición ante las pesquisas que se realicen en el nivel primario de atención de salud y rapidez para asistir al médico frente a la presencia de una señal atípica.

El nivel de atención de salud (tanto primaria, secundaria, como terciaria), es una fortaleza potencial para el Sistema Nacional de Salud (SNS) de Cuba, pero a la vez se torna débil ante el contexto económico actual del país, especialmente en relación al tratamiento del cáncer, donde los productos y terapéuticas disponibles son cada vez más costosos. Pueden señalarse igualmente, debilidades en el dominio y conocimiento del tema cáncer en los profesionales de la salud que laboran en los distintos niveles de atención, relevándose de forma marcada las insuficiencias del subsistema primario de salud, en tanto se refiere a oportunidades de servicios expeditos y efectivos.

El tratamiento mediático y público en términos de salud, ha sido como generalidad persistentemente fidedigno y respetuoso para con la sociedad y el sujeto social involucrado de modo directo. Sin embargo, en temas como el cáncer, donde aún en las propias instituciones hospitalarias es un tema tabú, este abordaje se proyecta en extremo conservador al tiempo que centralizado, con un lenguaje que no es todo lo directo que debiera ser, donde no se explotan los materiales testimoniales e historias de vida, lo que dificulta comprender por parte de los sujetos la dimensión de tal enfermedad y problematiza la asunción de un cambio de actitud ante la misma, tanto para el paciente como para la familia impactada.

En cuanto a la investigación científica y como parte de ella todo lo relativo a ensayos clínicos, ha de adquirir mayor nivel de socialización al menos en el sector de la salud, desde la perspectiva de una comunicación de la ciencia más horizontal a la sociedad en su conjunto, utilizando con mayor efectividad el valor de la información testimonial (pacientes, familiares) y de prescriptores (médicos, investigadores, analistas, administradores de salud). 
El Centro de Inmunología Molecular asume regularmente prácticas de divulgación científica de alto nivel, con énfasis en la diseminación de la información actualizada hacia su capital humano; de igual forma se proyecta hacia la comunidad médica y científica del sector, no siendo así para la comunidad o la sociedad en general. Aun cuando se expresa como una organización cuyos públicos internos están altamente sensibilizados y comprometidos con su misión y visión, desde una vocación humanista y ética, en sus plataformas misionales no queda del todo explícito su responsabilidad institucional para con la sociedad cubana en general, la ciudadanía, la comunidad, desde el concepto de proveedores de conocimiento, lo cual la comprensión y asunción de este horizonte de actuación.

El estudio permitió constatar asimismo, que los directivos y especialistas de CIM, sustentan una visión orgánica, sistémica, veraz y crítica que los convierte en agentes con formidables potencialidades para hacer transcender esa Responsabilidad Social Institucional con mucho mayor fuerza sobre la ciudadanía en su conjunto, sobre la comunidad más inmediata al centro y sobre el propio campo científico y asistencial al cual se vincula por su objeto social, su misión y su visión.

La pesquisa realizada igualmente concluye que se trata de una percepción pública compleja y diversa, hasta hoy estratificada y sectorializada; en formación, es decir no completamente configurada, en este sentido no es unívoca ni homogénea en torno a los actores concurrentes, en este punto por obvias razones la población, los pacientes, familiares y enfermos potenciales son los más vulnerables. Pese a ello, esta percepción en las condiciones actuales de la sociedad cubana sí es susceptible de ser pulsada y cubierta desde bases científicas, asistenciales y comunicativas, tomando a la comunicación para la salud, con sus múltiples herramientas y articulaciones posibles (información pública, promoción de salud, prevención, educación, responsabilidad social institucional), como plataforma de actuación para el cambio. 


\section{Referencias}

Anaya, B.; Martin, M. (2009). Biotecnología en Cuba: origen y resultados alcanzados. Centro de Estudios de la Economía Cubana, Universidad de La Habana.

Bastidas, N. (2012-2013). La mala práctica médica y los derechos humanos. Razón y Palabra, Primera Revista Electrónica en América Latina Especializada en Comunicación, Comunicación y Derechos Humanos, número 81.

Bourdieu, P. (s.f.). Algunas propiedades de los campos. Sociología y cultura, Editorial Grijalbo.

Cardoso, M.; Pereira, J. (2004). Comunicación, desarrollo y promoción de la salud. Enfoques, balances $y$ desafíos. Argentina. VIII Congreso Iberoamericano de la Comunicación y en VII Congreso Latinoamericano de Investigadores de la Comunicación, Universidad Nacional de la Plata.

Carullo, J. (2001). La percepción social de la ciencia y la tecnología: conceptos, metodologías de medición y ejemplos significativos. Argentina. Universidad Nacional de Quilmes.

Cavalli, F. (2012). Cáncer: El gran desafío. La Habana. Editorial Ciencias Médicas.

Chirinos, M.; Fernández, L.; Sánchez, G. (2012-2013). Responsabilidad empresarial o empresas socialmente responsables. Razón y Palabra, Primera Revista Electrónica en América Latina Especializada en Comunicación, Comunicación y Derechos Humanos, número 81.

Coe, G. Comunicación en Salud. Revista Chasqui, 63. Disponible en: http://chasqui.comunica.org/coe.htm [Consultado 12 de agosto, 2016]

Crombet, T. (2013) Entrevista inédita. La Habana. Centro de Inmunología Molecular.

de la Peña, J. A. (2005). La percepción pública de la ciencia en México. Ciencias, No. 078. Universidad Nacional Autónoma de México. Distrito Federal, México.

del Pino, T. (2010). La Comunicación Educativa para la Salud en la Atención Primaria. Un estudio de casos en Ciudad de La Habana. La Habana. Tesis de Maestría en Ciencias de la Comunicación, Facultad de Comunicación, Universidad de la Habana.

Díaz, H. (2012). La Comunicación en todas las políticas de salud. Revista de Comunicación y salud. Vol. 2, n 2, pp. 45-46.

Ferrer, A., León, G. (2013). Cultura científica y comunicación de la ciencia. México. Razón y Palabra, número 65.

García, A. La ética y al profesionalización en Educación para la salud. Revista Interuniversitaria Pedagogía Social "12-13", 33-42, Segunda época. ECSA NAULLIBRES.

García, E. (2011). Revolución y ciencia en Cuba: La Academia de Ciencia en Cuba 1962 1972. La Habana. Revista Anales de la Academia de Ciencias de Cuba, Vol. 1, No. 2.

Guerra, R., González, B., Valdés, D., Lage, A. (2012) Manual de organización y funciones del Centro de Inmunología Molecular. La Habana. Documento de política institucional del Centro de Inmunología Molecular. 
Jara, S.; Torres, J. (2011). Percepción social de la ciencia: ¿utopía o distopía? Argentina. Revista iberoamericana de ciencia tecnología y sociedad, vol.6 no.17.

Lage, A. (2007). Biotecnología en Cuba, Disponible en: http://www.profesionalespcm.org/ php/MuestraArticulo2.php?id=7932.

Lage, A. (2013). Entrevista inédita. La Habana. Centro de Inmunología Molecular.

Montes de Oca, J. L. (2011). La política científica y tecnológica de Cuba: Su impacto en el Sistema Nacional de Salud. Contribuciones a las Ciencias Sociales. Conferencia.

Osorio, M. (2013). Entrevista inédita. La Habana. Instituto Nacional de Oncología y Radiobiología.

Ríos, I. (2011). Comunicación en salud: conceptos y modelos teóricos. Chile. Perspectivas de la Comunicación, vol. 4, nº 1. ISSN 0718-4867, Universidad de la frontera.

Vallin, A. (2013). Entrevista inédita. La Habana. Centro de Inmunología Molecular.

\section{Agradecimientos}

Agradecemos al Centro de Inmunología Molecular y a los integrantes del Consejo de Dirección que formaron parte de la pesquisa.

A las instancias hospitalarias y médicos involucrados, por la colaboración ofrecida en el proceso de investigación: Hospital Hermanos Ameijeiras (HHAA); Instituto Nacional de Oncología y Radiobiología (INOR), y Hospital Centro de Investigaciones Médico Quirúrgicas (CIMEQ).

A los pacientes y familiares, que aún en las condiciones más difíciles brindaron total disposición y confianza para con el estudio. 